\title{
Allele and genotype frequencies of the SOD1 gene polymorphism associated with canine degenerative myelopathy in Belgian Malinois dogs in Greece
}

\author{
Antonia Mataragka ${ }^{1}$, John Ikonomopoulos ${ }^{1}$, Georgios S. Zervas ${ }^{1}$, Christos D. Vamvakidis ${ }^{2}$, Nikolaos Tzimotoudis ${ }^{3}$, \\ Ariadne Loukia Hager-Theodorides ${ }^{4}$, Maria Gazouli ${ }^{5}$ and Antonios Kominakis ${ }^{4}$
}

\begin{abstract}
1. School of Animal Biosciences, Department of Animal Science, Laboratory of Anatomy and Physiology of Farm Animals, Agricultural University of Athens, Athens, Greece; 2. Hellenic Army Biological Research Center/Veterinary Clinic, Athens, Greece; 3. Hellenic Army Biological Research Center/Laboratory of Microbiology, Athens, Greece; 4. School of Animal

Biosciences, Department of Animal Science, Laboratory of Animal Breeding and Husbandry, Agricultural University of

Athens, Athens, Greece; 5. School of Medicine, Department of Basic Medical Science, Laboratory of Biology, Kapodistrian University of Athens, Athens, Greece.
\end{abstract}

Corresponding author: Antonios Kominakis, e-mail: acom@aua.gr

Co-authors: AM: antonia.mataragka@gmail.com, JI: ikonomop@aua.gr; GSZ: zervasg95@gmail.com, CDV: vamchris@hotmail.com, NT: ntzimo@gmail.com, ALH: a.hager@aua.gr, MG: maria.gazouli@gmail.com.

Received: 12-11-2020, Accepted: 16-04-2021, Published online: 09-06-2021

doi: www.doi.org/10.14202/vetworld.2021.1472-1479 How to cite this article: Mataragka A, Ikonomopoulos J, Zervas GS, Vamvakidis CD, Tzimotoudis N, Hager-Theodorides AL, Gazouli M, Kominakis A (2021) Allele and genotype frequencies of the SOD1 gene polymorphism associated with canine degenerative myelopathy in Belgian Malinois dogs in Greece, Veterinary World, 14(6): 1472-1479.

\begin{abstract}
Background and Aim: Canine degenerative myelopathy (CDM) is an adult-onset fatal disorder associated with a point mutation of the superoxide dismutase 1 (SOD1) gene (SOD1:c.118G>A). This study aimed to determine the allele and genotype frequencies of this mutation in a group of Belgian Malinois dogs in Greece.

Materials and Methods: Samples $(n=72)$ of whole blood were collected from 72 purebred dogs of the Hellenic Armed Forces; these samples were processed for DNA isolation, polymerase chain reaction, and digestion with the restriction endonuclease AcuI. Sample testing was conducted in compliance with ISO17025 accreditation requirements.

Results: The observed relative genotype frequencies were $71 \%$ for the homozygous (GG), $25 \%$ for the heterozygous (AG), and $4 \%$ for the homozygous mutant (AA) alleles. These frequencies were close to those expected, indicating no significant departure from Hardy-Weinberg equilibrium (HWE, $\mathrm{p}=0.395$ ). The frequency of heterozygous animals indicates that a high risk of developing CDM in forthcoming generations exists in the tested population because mating among carriers would result in 25\% AA progeny. The medical record of the group of study animals indicated selection against leishmaniosis, as applied throughout generations by owners and breeders. The potential association of this selection with the HWE status of the study population was discussed.

Conclusion: The SOD1:c.118G>A mutation was common in the tested group of dogs; thus, they are suitable for a follow-up investigation on the development and progression of CDM. A case-control study on animals with evidence of sensitivity to infectious myelopathy could provide new insights into disease pathogenesis.
\end{abstract}

Keywords: degenerative myelopathy, dogs, genetic analysis, genetic polymorphism, restriction fragment length polymorphism-polymerase chain reaction, $S O D 1: c .118 \mathrm{~A}$.

\section{Introduction}

Canine degenerative myelopathy (CDM) is an adult-onset fatal neurodegenerative disorder characterized by progressive motor neuron loss and paralysis [1,2]. The clinical onset of the disease usually occurs in affected individuals aged $>8$ years, but definitive diagnosis is only possible postmortem [3]. CDM was initially described in German Shepherd dogs $[1,4,5]$ but has since been diagnosed in several other breeds of dog, including Pembroke Welsh Corgi,

Copyright: Mataragka, et al. Open Access. This article is distributed under the terms of the Creative Commons Attribution 4.0 International License (http://creativecommons.org/licenses/ by/4.0/), which permits unrestricted use, distribution, and reproduction in any medium, provided you give appropriate credit to the original author(s) and the source, provide a link to the Creative Commons license, and indicate if changes were made. The Creative Commons Public Domain Dedication waiver (http:// creativecommons.org/publicdomain/zero/1.0/) applies to the data made available in this article, unless otherwise stated.
Boxer, Chesapeake Bay Retriever, and Rhodesian Ridgeback [2,6-9].

$\mathrm{CDM}$ has been associated with a point mutation of the canine superoxide dismutase 1 (SOD1) gene (SOD1:c.118G>A) [2,7], which shows an autosomal recessive inheritance pattern with incomplete (reduced) penetrance [2]. Animals homozygous for the mutant allele (AA) have a strong predisposition for developing CDM [10], whereas carriers of the mutant allele, that is, heterozygotes (AG), are at low risk, probably because the disorder develops too slowly to become clinically apparent within the usual life span of a dog [2].

In humans, $S O D 1$ variants have been implicated in the pathogenesis of familial amyotrophic lateral sclerosis (ALS), a debilitating neurological disorder characterized by progressive degeneration of motor neurons; this disease is considered the human analog of CDM 
[11-13]. SOD1 is a cytosolic and mitochondrial antioxidant enzyme that protects cells from the toxicity of reactive oxygen species [14]. Mutant SOD1 tends to form intracellular aggregates due to misfolding, inducing accumulation in the affected motor neurons of another intracellular protein, namely, disulfide isomerase [15].

In the present study, we investigated the genetic basis of CDM in a dog breed, Belgian Malinois, with an increased predisposition to this disease, and a group of animals maintained under a strict program of health surveillance. The information provided was assessed with the goal of identifying risk factors potentially associated with CDM and ALS. This study aimed to determine the allele and genotype frequencies of the $S O D 1: c .118 G>A$ mutation in a group of Belgian Malinois and to compare the results with those previously recorded in relation to the same breed and other breeds.

\section{Materials and Methods}

\section{Ethical approval}

The biological material that was submitted to this investigation was collected exclusively for the purpose of the routine health monitoring of the animal subjects (service dogs of the Hellenic Armed Forces) and not for the purpose of this study. The latter did not involve handling of live of dead animals. According to the applicable legislation (Research Ethics Committee of the Agricultural University of Athens), ethics approval was not required for this study.

\section{Study period and location}

The study was conducted from July to October 2018. Samples $(n=72)$ of whole blood from 72 purebred Belgian Malinois dogs (one sample of 3-6 ml per animal) of the Hellenic Armed Forces were collected from Athens, Greece. The samples were processed at the Laboratory of Anatomy and Physiology of Farm Animals of the Agricultural University of Athens, Greece.

\section{Sample collection}

All animals were 2-7 years of age and healthy, based on a yearly assessment consisting of a clinical examination, biochemical blood analysis, and radiography of the thorax and limbs (which is primarily focused on assessing hip dysplasia). Blood samples were taken from the jugular vein and immediately divided into two portions, one of which was stored at $4-6^{\circ} \mathrm{C}$ for a maximum of $48 \mathrm{~h}$ until it was submitted for DNA isolation. The other portion was stored at $-20^{\circ} \mathrm{C}$ and used as stock.

\section{DNA isolation}

Blood samples were processed for DNA isolation using a commercially available kit and according to the manufacturer's instructions (NucleoSpin ${ }^{\circledR}$ Tissue, Macherey-Nagel GmbH and Co. KG, Germany). Isolated DNA was stored at $-20^{\circ} \mathrm{C}$ and then used for polymerase chain reaction (PCR). The quality of the isolated DNA was assessed in terms of its purity and integrity through agarose gel electrophoresis, followed by image analysis using a Bio-Rad ChemiDoc XRS+
Molecular Imager (Bio-Rad Laboratories Inc., USA), whereas spectrophotometry was used to measure optical density at 260/280 nm through a NanoDrop 8000 Spectrophotometer (Thermo Fisher Scientific Inc., USA). Fragmented DNA products were discarded, and DNA isolation was repeated from stock samples. DNA isolation and PCR were conducted in compliance with ISO17025 accreditation requirements.

\section{PCR-restriction fragment length polymorphism (RFLP) analysis}

PCR for the amplification of a 296-base pair (bp) fragment from the $S O D 1$ gene containing the targeted region (SOD1:c.118G>A) was conducted according to the previous methods [8] using the Invitrogen Taq DNA Polymerase Kit protocol (Thermo Fisher Scientific Inc.). The reaction mixture consisted of $1 \times$ PCR buffer, 0.75-U Taq DNA polymerase, $0.2 \mathrm{mM}$ dNTPs, $1.5 \mathrm{mM} \mathrm{MgCl}, 0.5 \mu \mathrm{M}$ of each of two primers (forward: 5'-AGTGGGCCTGTTGTGGTATC-3';

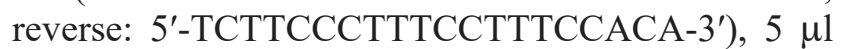
of DNA template, and PCR-grade water to a final volume of $50 \mu \mathrm{l}$. The thermal profile of the reaction was that proposed by Holder et al. [8]. PCR was conducted using an Applied Biosystems Verity 96-Well Thermal Cycler (Thermo Fisher Scientific Inc.).

PCR products were incubated at $37^{\circ} \mathrm{C}$ for $1 \mathrm{~h}$ with the restriction endonuclease $A c u I$ (New England Biolabs Inc., USA). The digestion products were submitted to agarose gel electrophoresis with a 100-bp molecular weight ladder (Nippon Genetics, Europe $\mathrm{GmbH}$ ) using 3\% ultrapure agarose gel (Thermo Fisher Scientific Inc.) stained with ethidium bromide $(0.5 \mu \mathrm{g} / \mathrm{mL})$. The results were assessed using a BioRad ChemiDoc XRS+ Molecular Imager (Bio-Rad Laboratories Inc.).

For specificity confirmation, approximately $20 \%$ of the PCR-RFLP products were submitted for sequence analysis, which was conducted on both strands using the Applied Biosystems BigDye Terminator Cycle Sequencing Kit and a PRISM 377 DNA Sequencer (Thermo Fisher Scientific Inc.). The results were compared against deposited sequences in the GenBank database using Basic Local Alignment Search Tool from the National Center for Biotechnology Information.

\section{Genetic analysis}

Allele and genotype frequencies were estimated using the Genepop (online ver. 4.7) software (Michel Raymond and Francois Rousset, Laboratiore de Genetique et Environment, Montpellier, France) [16], which was also used to conduct an exact probability test for Hardy-Weinberg equilibrium (HWE) using the Markov chain method. The assessment of the $\mathrm{F}_{\text {IS }}$ fixation index was conducted as previously described [17]. $\mathrm{F}_{\text {IS }}$ is a measure of the average departure of the observed from the expected (under the assumption of HWE) frequencies with theoretical values ranging from -1.0 (all individuals heterozygous or 
entirely outbred) to +1.0 (no observed heterozygotes or entirely inbred).

\section{Results}

An AcuI restriction site (CTGAAG(N) $\left.{ }_{16} \downarrow\right)$ is present in the PCR product corresponding to the wildtype SOD1:c.118G allele; hence, restriction analysis generates two DNA fragments (230 and $62 \mathrm{bp}$ ) in homozygous GG animals. The mutant allele does not present the Acul restriction site; it generates one fragment of 292 bp in homozygous AA animals but three fragments $(292,230$, and $62 \mathrm{bp})$ in heterozygous (GA) animals (Figure-1). The frequency of the $\mathrm{G}$ and A alleles was 0.833 and 0.167 , respectively.

The observed and expected (under the assumption of HWE) frequencies of the three genotypes are shown in Table-1. The observed relative genotype frequencies were $71 \%(\mathrm{n}=51)$ for the homozygous $(\mathrm{GG})$, $25 \%(n=18)$ for the heterozygous (AG), and $4 \%(n=3)$ for the homozygous mutant (AA) alleles. These frequencies were close to those expected for the three genotypes $(\mathrm{GG}=69 \%$; $\mathrm{GA}=20 \%$; and $\mathrm{AA}=3 \%$ ), indicating no significant departure from $\operatorname{HWE}(p=0.395)$. For the locus under study, HWE was also confirmed by the low positive value of the $\mathrm{F}_{\text {IS }}$ statistic (0.107), which indicated a low heterozygote deficit (18 heterozygous animals observed vs. 20 expected) (Table-1).

\section{Discussion}

In the studied group of animals, the frequency of the mutant allele (A) was 0.17 , which is slightly higher than that previously reported for Belgian Malinois $(0-0.14)[9,18-20]$ but similar to the frequency reported in other breeds such as the Airedale Terrier, Australian Terrier, Chinese Crested, Chow Chow, French Bulldog, Irish Setter, and Puli $[9,18]$ (Table-2).

Table-1: Number of observed and expected genotypes (relative frequencies are reported in parenthesis).

\begin{tabular}{lcc}
\hline Genotype & Observed & Expected \\
\hline GG & $51(0.71)$ & $50(0.69)$ \\
GA & $18(0.25)$ & $20(0.28)$ \\
AA & $3(0.04)$ & $2(0.03)$ \\
Total & $72(100)$ & $72(100)$ \\
\hline
\end{tabular}

The heterozygosity and homozygosity rates observed were similar to those previously recorded in the same breed $(0.25$ vs. $0-0.17$ and 0.04 vs. $0-0.06$ for heterozygosity and homozygosity, respectively) $[9,18-20]$ and other breeds (Airedale Terrier, American Hairless Terrier, Beagle, Belgian Sheepdog, Borzoi, Boxer, Boykin, Chow Chow, Spaniel, and Kuvasz) (Table-2) [6-9,18-24].

The appreciable frequency of heterozygous (carriers) animals indicates that selective breeding is necessary to reduce the high risk of developing CDM in forthcoming generations of this Belgian Malinois population: Mating among carriers would result in $25 \%$ AA progeny. However, genetic selection using the SOD1:c.118G>A mutation against CDM must be carefully conducted to avoiding genetic erosion caused by inbreeding and the reduction of the effective population size. Notably, the management practice applied to the studied group of animals by the Hellenic Armed Forces aimed to avoid inbreeding, mainly by selecting individuals from different breeders in Greece based on breed standards and pedigree records.

Medical records were analyzed, and it was noteworthy that the targeted population had been maintained under a "test and remove" practice against leishmaniosis; this practice had been applied across generations by breeders and the Hellenic Army. The fact that the tested population was under HWE may be associated with the impact of this selection criterion on the genetic constitution of the population, particularly the frequency of the mutant allele [25]. This is consistent with the role of the wild-type SOD1 gene in building a strong immune response against intracellular pathogens such as Leishmania spp. [26-29]. Thus, the removal of Leishmania-positive individuals from the parental population of the tested animals for several generations may have decreased the frequency of the mutant allele to the level observed in this study. Notably, the link between DM and infectious myelopathy, a typical feature of canine leishmaniosis, is also supported by the association of pathogenesis in CDManalogous diseases of cats and humans, that is, feline DM and ALS, with the feline leukemia virus [30-32] and retrovirus infections, respectively $[11,13]$.

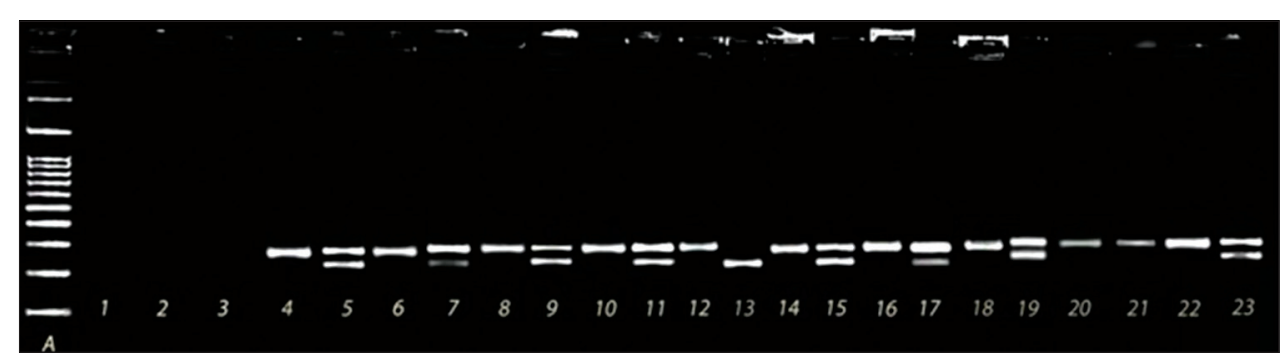

Figure-1: Agarose gel electrophoresis of the polymerase chain reaction (PCR) and restriction fragment length polymorphism (RFLP) products of representative samples tested for the detection of the (SOD1:c.118G>A) gene mutation. Lane A. DNA 100 bp Ladder (Nippon Genetics, Europe $\mathrm{GmbH}$ ). Lanes 1-3. Negative controls (samples containing water instead of DNA) for DNA isolation (lane 1), PCR (lane 2), and RFLP-PCR (lane 3). Lanes 4, 6, 8, 10, 12, 14, 16, 18, 20, and 22. PCR amplification products of the SOD1 gene. Lanes $5,7,9,11,13,15,17,19,21$, and 23 . The outcome of RFLP analysis conducted on PCR products using the restriction endonuclease AcuI for the GA genotype (lanes 5, 7, 9, 11, 15, 17, 19, and 23), the GG genotype (lane 13), and the AA genotype (lane 21). 
Table-2: Allele (A) and genotype frequencies of polymorphisms of the SOD1 gene across various canine breeds.

\begin{tabular}{|c|c|c|c|c|c|}
\hline \multirow[t]{2}{*}{ Breed or variety } & \multicolumn{3}{|c|}{ Genotype frequency } & \multirow[t]{2}{*}{ A allele frequency } & \multirow[t]{2}{*}{ Reference } \\
\hline & GG & GA & AA & & \\
\hline Affenpinscher & 1.00 & 0.00 & 0.00 & 0.00 & [9] \\
\hline Afghan Hound & 1.00 & 0.00 & 0.00 & 0.00 & [9] \\
\hline Airedale Terrier & $0.50-0.70$ & $0.25-0.30$ & $0.00-0.25$ & $0.15-0.38$ & {$[9,18]$} \\
\hline Akbash Dog & 1.00 & 0.00 & 0.00 & 0.00 & [9] \\
\hline Akita & 1.00 & 0.00 & 0.00 & 0.00 & {$[9,18]$} \\
\hline Alaskan Husky & 1.00 & 0.00 & 0.00 & 0.00 & {$[9,18]$} \\
\hline Alaskan Klee Kai & 1.00 & 0.00 & 0.00 & 0.00 & [9] \\
\hline Alaskan Malamute & $0.98-1.00$ & 0.00 & $0.00-0.02$ & $0.00-0.02$ & {$[9,18]$} \\
\hline Alaskan Noble Companion Dog & 1.00 & 0.00 & 0.00 & 0.00 & [9] \\
\hline American Bulldog & $0.92-1.00$ & $0.00-0.05$ & $0.00-0.03$ & $0.00-0.05$ & {$[9,18]$} \\
\hline American Eskimo Dog & $0.30-0.33$ & $0.35-0.60$ & $0.10-0.33$ & $0.40-0.50$ & {$[9,18]$} \\
\hline American Foxhound & $0.50-1.00$ & $0.00-0.5$ & 0.00 & $0.00-0.25$ & {$[9,18]$} \\
\hline American Hairless (Rat) Terrier & 0.75 & 0.25 & 0.00 & 0.13 & [9] \\
\hline American Staffordshire Terrier & $0.63-1.00$ & $0.00-0.08$ & $0.00-0.29$ & $0.00-0.33$ & {$[9,18,21]$} \\
\hline American Water Spaniel & $0.24-0.32$ & $0.45-0.51$ & $0.23-0.25$ & $0.46-0.51$ & {$[9,18]$} \\
\hline Anatolian Shepherd Dog & 0.83 & 0.13 & 0.03 & 0.10 & [9] \\
\hline Argentine Dogo & 1.00 & 0.00 & 0.00 & 0.00 & [9] \\
\hline Australian Cattle Dog & $0.87-0.89$ & $0.08-0.11$ & $0.00-0.05$ & $0.05-0.09$ & {$[9,18]$} \\
\hline Australian Kelpie & 0.00 & 0.50 & 0.50 & 0.75 & [9] \\
\hline Australian Shepherd & $0.00-0.79$ & $0.18-1.00$ & $0.00-0.32$ & $0.12-0.50$ & {$[9,18,20]$} \\
\hline Australian Terrier & $0.50-0.67$ & $0.33-0.50$ & 0.00 & $0.17-0.25$ & {$[9,18]$} \\
\hline Basenji & 1.00 & 0.00 & 0.00 & 0.00 & {$[9,18]$} \\
\hline Basset Hound & 1.00 & 0.00 & 0.00 & 0.00 & {$[9,18]$} \\
\hline Beagle & $0.90-0.91$ & $0.05-0.10$ & $0.00-0.04$ & $0.05-0.06$ & {$[9,18]$} \\
\hline Bearded Collie & 1.00 & 0.00 & 0.00 & 0.00 & {$[9,18]$} \\
\hline Beauceron & 1.00 & 0.00 & 0.00 & 0.00 & {$[9,18]$} \\
\hline Bedlington Terrier & 1.00 & 0.00 & 0.00 & 0.00 & {$[9,18]$} \\
\hline Belgian Malinois & $0.78-1.00$ & $0.00-0.17$ & $0.00-0.06$ & $0.00-0.14$ & {$[9,18-20]$} \\
\hline Belgian Sheepdog & $0.75-0.79$ & $0.11-0.25$ & $0.00-0.11$ & $0.12-0.16$ & {$[9,18]$} \\
\hline Belgian Tervuren & 0.94 & 0.04 & 0.02 & 0.04 & [9] \\
\hline Bergamasco & 1.00 & 0.00 & 0.00 & 0.00 & [9] \\
\hline Berger Picard & 1.00 & 0.00 & 0.00 & 0.00 & [9] \\
\hline Bernese Mountain Dog & $0.37-0.60$ & $0.35-0.46$ & $0.05-0.18$ & $0.23-0.41$ & {$[9,18,21]$} \\
\hline Bichon Frise & $0.96-1.00$ & 0.00 & $0.00-0.04$ & $0.00-0.04$ & {$[9,18]$} \\
\hline Black \& Tan Coonhound & 1.00 & 0.00 & 0.00 & 0.00 & [9] \\
\hline Black Russian Terrier & 1.00 & 0.00 & 0.00 & 0.00 & [9] \\
\hline Bloodhound & $0.47-0.50$ & 0.44 & $0.06-0.08$ & $0.28-0.30$ & {$[9,18]$} \\
\hline Blue Heeler & 1.00 & 0.00 & 0.00 & 0.00 & [9] \\
\hline Bluetick Coonhound & 0.75 & 0.00 & 0.25 & 0.25 & [9] \\
\hline Boerboel & 1.00 & 0.00 & 0.00 & 0.00 & [9] \\
\hline Border Collie & $0.79-0.99$ & $0.01-0.09$ & $0.00-0.13$ & $0.008-0.17$ & {$[9,18,20-22]$} \\
\hline Border Terrier & $0.97-1.00$ & 0.00 & $0.00-0.03$ & $0.00-0.03$ & {$[9,18]$} \\
\hline Borzoi & $0.70-1.00$ & $0.00-0.27$ & $0.00-0.04$ & $0.00-0.17$ & {$[9,18]$} \\
\hline Boston Terrier & $0.93-1.00$ & 0.00 & $0.00-0.07$ & $0.00-0.07$ & {$[9,18]$} \\
\hline Bouvier des Flandres & 1.00 & 0.00 & 0.00 & 0.00 & {$[9,18]$} \\
\hline Boxer & $0.12-0.87$ & $0.13-0.34$ & $0.00-0.57$ & $0.07-0.72$ & {$[9,18,20,21]$} \\
\hline Boykin Spaniel & 0.75 & 0.25 & 0.00 & 0.13 & [9] \\
\hline Briard & 1.00 & 0.00 & 0.00 & 0.00 & {$[9,18]$} \\
\hline Brittany & 1.00 & 0.00 & 0.00 & 0.00 & {$[9,18]$} \\
\hline Brussels Griffon & 1.00 & 0.00 & 0.00 & 0.00 & [9] \\
\hline Bulldog & 0.46 & 0.54 & 0.00 & 0.27 & [9] \\
\hline Bullmastiff & 0.92 & 0.00 & 0.08 & 0.08 & [9] \\
\hline Bull Terrier & 1.00 & 0.00 & 0.00 & 0.00 & {$[9,18]$} \\
\hline Cairn Terrier & 1.00 & 0.00 & 0.00 & 0.00 & {$[9,18]$} \\
\hline Canaan Dog & $0.45-0.51$ & $0.40-0.44$ & $0.09-0.11$ & $0.29-0.33$ & {$[9,18]$} \\
\hline Cane Corso & 1.00 & 0.00 & 0.00 & 0.00 & [9] \\
\hline Cardigan Welsh Corgi & $0.46-0.50$ & $0.36-0.44$ & $0.10-0.15$ & 0.32 & {$[9,18]$} \\
\hline Catahoula Leopard Dog & 0.50 & 0.50 & 0.00 & 0.25 & [9] \\
\hline Cavalier King Charles Spaniel & $0.09-0.14$ & $0.37-0.82$ & $0.09-0.49$ & $0.50-0.68$ & {$[9,18]$} \\
\hline Central Asian Sheepdog (Ovcharka) & 1.00 & 0.00 & 0.00 & 0.00 & [9] \\
\hline Chesapeake Bay Retriever & $0.34-0.41$ & $0.42-0.44$ & $0.15-0.24$ & $0.37-0.45$ & {$[9,18]$} \\
\hline Chihuahua & 1.00 & 0.00 & 0.00 & 0.00 & [9] \\
\hline Chinese Crested & $0.63-0.75$ & $0.25-0.37$ & 0.00 & $0.12-0.18$ & {$[9,18]$} \\
\hline Chinese Shar Pei & 1.00 & 0.00 & 0.00 & 0.00 & {$[9,18]$} \\
\hline \multirow[t]{2}{*}{ Chinook } & 1.00 & 0.00 & 0.00 & 0.00 & {$[9,18]$} \\
\hline & & & & & $($ Contd...) \\
\hline
\end{tabular}


Table-2: (Continued).

\begin{tabular}{|c|c|c|c|c|c|}
\hline \multirow[t]{2}{*}{ Breed or variety } & \multicolumn{3}{|c|}{ Genotype frequency } & \multirow[t]{2}{*}{ A allele frequency } & \multirow[t]{2}{*}{ Reference } \\
\hline & GG & GA & AA & & \\
\hline Chow Chow & $0.64-0.70$ & $0.27-0.32$ & $0.03-0.05$ & $0.16-0.20$ & {$[9,18]$} \\
\hline Clumber Spaniel & 0.91 & 0.09 & 0.00 & 0.05 & {$[9,18]$} \\
\hline Cocker Spaniel (American) & $0.97-1.00$ & $0.00-0.03$ & 0.00 & $0.00-0.01$ & {$[9,18]$} \\
\hline Collie & $0.48-0.76$ & $0.24-0.28$ & $0.00-0.26$ & $0.12-0.39$ & {$[9,18,23]$} \\
\hline Coton de Tulear & $0.85-0.87$ & $0.13-0.15$ & 0.00 & 0.07 & {$[9,18]$} \\
\hline Curly Coated Retriever & 1.00 & 0.00 & 0.00 & 0.00 & {$[9,18]$} \\
\hline Czechoslovakian Wolfdog & $0.46-0.54$ & $0.40-0.43$ & $0.04-0.13$ & $0.25-0.34$ & {$[9,24]$} \\
\hline Dachshund & 1.00 & 0.00 & 0.00 & 0.00 & {$[9,18]$} \\
\hline Dalmatian & $0.96-0.97$ & $0.02-0.03$ & $0.00-0.02$ & $0.03-0.15$ & {$[9,18]$} \\
\hline Dandie Dinmont Terrier & 1.00 & 0.00 & 0.00 & 0.00 & {$[9,18]$} \\
\hline Decker Terrier & 0.88 & 0.13 & 0.00 & 0.06 & [9] \\
\hline Doberman Pinscher & $0.98-1.00$ & 0.00 & $0.00-0.02$ & $0.00-0.02$ & {$[9,18]$} \\
\hline Dogue du Bordeaux & 1.00 & 0.00 & 0.00 & 0.00 & {$[9,18]$} \\
\hline Dutch Shepherd & $0.00-0.83$ & $0.17-1.00$ & 0.00 & $0.08-0.50$ & {$[9,18]$} \\
\hline English Bulldog & 0.83 & 0.17 & 0.00 & 0.08 & {$[20]$} \\
\hline English Cocker Spaniel & 1.00 & 0.00 & 0.00 & 0.00 & {$[9,18]$} \\
\hline English Coonhound & 0.88 & 0.13 & 0.00 & 0.06 & [9] \\
\hline English Foxhound & 1.00 & 0.00 & 0.00 & 0.00 & {$[9,18]$} \\
\hline English Setter & 1.00 & 0.00 & 0.00 & 0.00 & {$[9,18]$} \\
\hline English Shepherd & $0.89-0.90$ & $0.10-0.11$ & 0.00 & $0.05-0.06$ & {$[9,18]$} \\
\hline English Springer Spaniel & $0.76-0.78$ & $0.13-0.19$ & $0.03-0.10$ & $0.12-0.17$ & {$[9,18]$} \\
\hline English Toy Spaniel & 0.59 & 0.38 & 0.03 & 0.22 & [9] \\
\hline Field Spaniel & 1.00 & 0.00 & 0.00 & 0.00 & {$[9,18]$} \\
\hline Finnish Lapphund & 0.87 & 0.13 & 0.00 & 0.06 & {$[9,18]$} \\
\hline Finnish Spitz & 0.97 & 0.03 & 0.00 & 0.02 & {$[9,18]$} \\
\hline Flat-Coated Retriever & $0.92-1.00$ & $0.00-0.05$ & $0.00-0.03$ & $0.00-0.05$ & {$[9,18]$} \\
\hline Fox Terrier-Smooth & 1.00 & 0.00 & 0.00 & 0.00 & {$[9,18]$} \\
\hline Fox Terrier-Wire & $0.01-0.03$ & $0.09-0.15$ & $0.82-0.90$ & $0.90-0.94$ & {$[9,18]$} \\
\hline French Bulldog & $064-0.74$ & $0.21-0.27$ & $0.06-0.09$ & $0.16-0.23$ & {$[9,18]$} \\
\hline German Pinscher & 0.93 & 0.07 & 0.00 & 0.03 & {$[9,18]$} \\
\hline German Shepherd Dog & $0.44-0.81$ & $0.13-0.37$ & $0.03-0.22$ & $0.13-0.38$ & {$[7-9,18-21]$} \\
\hline German Shorthaired Pointer & $0.91-1.00$ & $0.00-0.02$ & $0.00-0.07$ & $0.00-0.08$ & {$[9,18]$} \\
\hline German Wirehaired Pointer & 0.91 & 0.09 & 0.00 & 0.05 & [9] \\
\hline Giant Schnauzer & $0.98-1.00$ & 0.00 & $0.00-0.02$ & $0.00-0.02$ & {$[9,18]$} \\
\hline Glen of Imaal Terrier & 1.00 & 0.00 & 0.00 & 0.00 & [9] \\
\hline Golden Retriever & $0.96-1.00$ & $0.00-0.01$ & $0.00-0.03$ & $0.00-0.03$ & {$[9,18,21]$} \\
\hline Gordon Setter & $0.98-1.00$ & 0.00 & $0.00-0.03$ & $0.00-0.03$ & {$[9,18]$} \\
\hline Great Dane & 1.00 & 0.00 & 0.00 & 0.00 & {$[9,18]$} \\
\hline Great Pyrenees & $0.80-0.81$ & $0.12-0.15$ & $0.04-0.08$ & $0.12-0.14$ & {$[9,18]$} \\
\hline Greater Swiss Mountain Dog & 1.00 & 0.00 & 0.00 & 0.00 & {$[9,18]$} \\
\hline Greyhound & 0.97 & 0.00 & 0.03 & 0.03 & [9] \\
\hline Hanoverian Hound & 1.00 & 0.00 & 0.00 & 0.00 & [9] \\
\hline Harrier & $0.81-0.86$ & 0.14-0.19 & 0.00 & $0.07-0.10$ & {$[9,18]$} \\
\hline Havana Silk Dog & 1.00 & 0.00 & 0.00 & 0.00 & [9] \\
\hline Havanese & 1.00 & 0.00 & 0.00 & 0.00 & {$[9,18]$} \\
\hline Hovawart & 0.48 & 0.27 & 0.25 & 0.38 & [9] \\
\hline Ibizan Hound & 1.00 & 0.00 & 0.00 & 0.00 & {$[9,18]$} \\
\hline Icelandic Sheepdog & 1.00 & 0.00 & 0.00 & 0.00 & {$[9,18]$} \\
\hline Irish Red and White Setter & 1.00 & 0.00 & 0.00 & 0.00 & {$[9,18]$} \\
\hline Irish Setter & $0.68-0.74$ & $0.21-0.29$ & $0.03-0.05$ & $0.16-0.18$ & {$[9,18]$} \\
\hline Irish Terrier & 0.90 & 0.10 & 0.00 & 0.05 & {$[9,18]$} \\
\hline Irish Water Spaniel & 1.00 & 0.00 & 0.00 & 0.00 & {$[9,18]$} \\
\hline Irish Wolfhound & $0.93-0.94$ & $0.06-0.07$ & 0.00 & 0.03 & {$[9,18]$} \\
\hline Italian Greyhound & 1.00 & 0.00 & 0.00 & 0.00 & {$[9,18]$} \\
\hline Jack Russell Terrier & $0.60-0.78$ & $0.17-0.19$ & $0.04-0.23$ & $0.13-0.32$ & {$[9,18]$} \\
\hline Japanese Chin & 1.00 & 0.00 & 0.00 & 0.00 & {$[9,18]$} \\
\hline Karelian Bear Dog & 1.00 & 0.00 & 0.00 & 0.00 & [9] \\
\hline Keeshond & $0.97-0.98$ & $0.02-0.03$ & 0.00 & $0.01-0.02$ & {$[9,18]$} \\
\hline Kerry Blue Terrier & 0.46 & $0.35-0.40$ & $0.15-0.19$ & $0.34-0.37$ & {$[9,18]$} \\
\hline King Shepherd & 0.56 & 0.28 & 0.17 & 0.31 & [9] \\
\hline Komondor & 0.58 & 0.40 & 0.02 & 0.22 & [9] \\
\hline Kuvasz & $0.61-0.65$ & $0.25-0.27$ & $0.08-0.13$ & $0.22-0.26$ & {$[9,18]$} \\
\hline Labrador Retriever & $0.91-1.00$ & $0.00-0.04$ & $0.00-0.05$ & $0.00-0.07$ & {$[9,18,21]$} \\
\hline Leonberger & 1.00 & 0.00 & 0.00 & 0.00 & {$[9,18]$} \\
\hline \multirow[t]{2}{*}{ Lhasa Apso } & 1.00 & 0.00 & 0.00 & 0.00 & {$[9,18]$} \\
\hline & & & & & (Contd... \\
\hline
\end{tabular}


Table-2: (Continued).

\begin{tabular}{|c|c|c|c|c|c|}
\hline \multirow[t]{2}{*}{ Breed or variety } & \multicolumn{3}{|c|}{ Genotype frequency } & \multirow[t]{2}{*}{ A allele frequency } & \multirow[t]{2}{*}{ Reference } \\
\hline & GG & GA & AA & & \\
\hline Lowchen & 1.00 & 0.00 & 0.00 & 0.00 & {$[9,18]$} \\
\hline Maltese & 1.00 & 0.00 & 0.00 & 0.00 & [9] \\
\hline Manchester Terrier-Standard & 1.00 & 0.00 & 0.00 & 0.00 & {$[9,18]$} \\
\hline Manchester Terrier-Toy & 1.00 & 0.00 & 0.00 & 0.00 & {$[9,18]$} \\
\hline Maremma & 0.50 & 0.00 & 0.50 & 0.50 & [9] \\
\hline Mastiff (English Mastiff) & $0.77-0.82$ & $0.17-0.19$ & $0.02-0.03$ & $0.10-0.13$ & {$[9,18]$} \\
\hline Miniature Bull Terrier & 1.00 & 0.00 & 0.00 & 0.00 & {$[9,18]$} \\
\hline Miniature Pinscher & 1.00 & 0.00 & 0.00 & 0.00 & [9] \\
\hline Miniature Schnauzer & $0.96-1.00$ & 0.00 & $0.00-0.04$ & $0.00-0.04$ & {$[9,18]$} \\
\hline Mountain Cur & 0.00 & 0.00 & 1.00 & 1.00 & [9] \\
\hline Mudi & 1.00 & 0.00 & 0.00 & 0.00 & [9] \\
\hline Native Am Indian Dog & 1.00 & 0.00 & 0.00 & 0.00 & [9] \\
\hline Neapolitan Mastiff & 1.00 & 0.00 & 0.00 & 0.00 & {$[9,18]$} \\
\hline Newfoundland & $0.98-1.00$ & 0.00 & $0.00-0.02$ & $0.00-0.02$ & {$[9,18]$} \\
\hline Norwegian Buhund & 1.00 & 0.00 & 0.00 & 0.00 & [9] \\
\hline Norwegian Elkhound & 1.00 & 0.00 & 0.00 & 0.00 & [9] \\
\hline Norwegian Lundehund & 1.00 & 0.00 & 0.00 & 0.00 & {$[9,18]$} \\
\hline Norfolk Terrier & 0.00 & 0.67 & 0.33 & 0.67 & [9] \\
\hline Norwich Terrier & $0.81-1.00$ & $0.00-0.19$ & 0.00 & $0.00-0.09$ & {$[9,18]$} \\
\hline Nova Scotia Duck Tolling Retriever & $0.78-0.88$ & $0.10-0.19$ & $0.02-0.03$ & $0.07-0.13$ & {$[9,18]$} \\
\hline Olde English Bulldogge & 0.50 & 0.25 & 0.25 & 0.38 & [9] \\
\hline Old English Sheepdog & $0.89-1.00$ & $0.00-0.11$ & 0.00 & $0.00-0.06$ & {$[9,18,20]$} \\
\hline Otterhound & 1.00 & 0.00 & 0.00 & 0.00 & {$[9,18]$} \\
\hline Papillon & 1.00 & 0.00 & 0.00 & 0.00 & {$[9,18]$} \\
\hline Parson Russell Terrier & 1.00 & 0.00 & 0.00 & 0.00 & {$[9,18]$} \\
\hline Patterdale Terrier & 0.00 & 0.00 & 1.00 & 1.00 & [9] \\
\hline Pembroke Welsh Corgi & $0.00-0.09$ & $0.28-0.43$ & $0.48-0.68$ & $0.697-0.83$ & {$[6,9,18,20]$} \\
\hline Perro de Presa Canario & 1.00 & 0.00 & 0.00 & 0.00 & [9] \\
\hline Peruvian Inca Orchid & 0.00 & 0.00 & 1.00 & 1.00 & [9] \\
\hline Petit Basset Griffon Vendeen & 1.00 & 0.00 & 0.00 & 0.00 & {$[9,18]$} \\
\hline Pharaoh Hound & $0.97-1.00$ & 0.00 & $0.00-0.03$ & $0.00-0.03$ & {$[9,18]$} \\
\hline Pit Bull Terrier & 0.43 & 0.11 & 0.45 & 0.51 & [9] \\
\hline Plott & 0.96 & 0.04 & 0.00 & 0.02 & [9] \\
\hline Pointer & $0.92-1.00$ & 0.00 & $0.00-0.08$ & $0.00-0.08$ & {$[9,18]$} \\
\hline Polish Lowland Sheepdog & 1.00 & 0.00 & 0.00 & 0.00 & [9] \\
\hline Pomeranian & $0.76-0.79$ & $0.18-0.21$ & 0.03 & $0.12-0.14$ & {$[9,18]$} \\
\hline Poodle-Miniature & 0.93 & 0.07 & 0.00 & 0.04 & [9] \\
\hline Poodle-Standard & $0.88-0.91$ & $0.06-0.12$ & $0.00-0.03$ & $0.06-0.07$ & {$[9,18,20]$} \\
\hline Poodle-Toy & 0.75 & 0.25 & 0.00 & 0.13 & [9] \\
\hline Portuguese Podengo & 0.97 & 0.03 & 0.00 & 0.02 & [9] \\
\hline Portuguese Pointer & 1.00 & 0.00 & 0.00 & 0.00 & [9] \\
\hline Portuguese Water Dog & 1.00 & 0.00 & 0.00 & 0.00 & {$[9,18]$} \\
\hline Pug & $0.46-0.67$ & $0.30-0.33$ & $0.00-0.23$ & $0.17-0.38$ & {$[9,18,20]$} \\
\hline Puli & 0.71 & 0.24 & 0.06 & 0.17 & [9] \\
\hline Pumi & 0.88 & 0.13 & 0.00 & 0.06 & [9] \\
\hline Pyrenean Shepherd & 1.00 & 0.00 & 0.00 & 0.00 & {$[9,18]$} \\
\hline Rat Terrier & 0.98 & 0.02 & 0.00 & 0.01 & [9] \\
\hline Redbone Coonhound & 1.00 & 0.00 & 0.00 & 0.00 & [9] \\
\hline Rhodesian Ridgeback & $0.46-0.52$ & $0.39-0.42$ & $0.09-0.11$ & $0.28-0.33$ & {$[9,18]$} \\
\hline Romanian Mioritic Shepherd & 1.00 & 0.00 & 0.00 & 0.00 & [9] \\
\hline Rottweiler & $0.86-0.96$ & $0.03-0.14$ & $0.00-0.01$ & $0.02-0.07$ & {$[9,18,20]$} \\
\hline Russell Terrier & 0.75 & 0.25 & 0.00 & 0.13 & [9] \\
\hline Saint Bernard & $0.77-1.00$ & $0.00-0.19$ & $0.00-0.04$ & $0.00-0.13$ & {$[9,18]$} \\
\hline Saluki & $0.92-1.00$ & $0.00-0.04$ & $0.00-0.04$ & $0.00-0.06$ & {$[9,18]$} \\
\hline Samoyed & $0.98-1.00$ & $0.00-0.02$ & 0.00 & $0.00-0.01$ & {$[9,18]$} \\
\hline Sapsaree & 1.00 & 0.00 & 0.00 & 0.00 & [9] \\
\hline Schipperke & 1.00 & 0.00 & 0.00 & 0.00 & {$[9,18]$} \\
\hline Scottish Deerhound & 1.00 & 0.00 & 0.00 & 0.00 & {$[9,18]$} \\
\hline Scottish Terrier & 1.00 & 0.00 & 0.00 & 0.00 & {$[9,18]$} \\
\hline Sealyham Terrier & $0.70-0.71$ & $0.29-0.30$ & 0.00 & 0.15 & {$[9,18]$} \\
\hline Shetland Sheepdog & $0.71-0.83$ & 0.17 & $0.00-0.12$ & $0.09-0.21$ & {$[9,18]$} \\
\hline Shiba Inu & 1.00 & 0.00 & 0.00 & 0.00 & {$[9,18]$} \\
\hline Shih Tzu & $0.38-0.43$ & $0.29-0.50$ & $0.13-0.29$ & $0.09-0.43$ & {$[9,18]$} \\
\hline Shiloh Shepherd & 0.73 & 0.24 & 0.03 & 0.15 & [9] \\
\hline \multirow[t]{2}{*}{ Siberian Husky } & $0.95-1.00$ & $0.00-0.03$ & $0.00-0.02$ & $0.00-0.04$ & {$[9,18]$} \\
\hline & & & & & $($ Contd...) \\
\hline
\end{tabular}


Table-2: (Continued).

\begin{tabular}{|c|c|c|c|c|c|}
\hline \multirow[t]{2}{*}{ Breed or variety } & \multicolumn{3}{|c|}{ Genotype frequency } & \multirow[t]{2}{*}{ A allele frequency } & \multirow[t]{2}{*}{ Reference } \\
\hline & GG & GA & AA & & \\
\hline Siberian Laika & 0.00 & 1.00 & 0.00 & 0.50 & [9] \\
\hline Silken Windhound & 1.00 & 0.00 & 0.00 & 0.00 & [9] \\
\hline Silky Terrier & 0.74 & 0.16 & 0.10 & 0.18 & [9] \\
\hline Skye Terrier & 1.00 & 0.00 & 0.00 & 0.00 & [9] \\
\hline Small Munsterlander & 1.00 & 0.00 & 0.00 & 0.00 & [9] \\
\hline Soft Coated Wheaten Terrier & $0.51-0.83$ & $0.17-0.20$ & $0.00-0.28$ & $0.09-0.39$ & {$[9,18]$} \\
\hline Spinone Italiano & 1.00 & 0.00 & 0.00 & 0.00 & {$[9,18]$} \\
\hline Staffordshire Bull Terrier & $0.88-0.94$ & $0.00-0.02$ & $0.06-0.10$ & $0.06-0.11$ & {$[9,18]$} \\
\hline Standard Schnauzer & $0.97-1.00$ & $0.00-0.03$ & 0.00 & $0.00-0.01$ & {$[9,18]$} \\
\hline Sussex Spaniel & 1.00 & 0.00 & 0.00 & 0.00 & {$[9,18]$} \\
\hline Swedish Valhund & 1.00 & 0.00 & 0.00 & 0.00 & {$[9,18]$} \\
\hline Tamaskan (Aatu Tamaskan) & 0.68 & 0.29 & 0.03 & 0.18 & [9] \\
\hline Tenterfield Terrier & 0.89 & 0.11 & 0.00 & 0.05 & [9] \\
\hline Tibetan Mastiff & 1.00 & 0.00 & 0.00 & 0.00 & {$[9,18]$} \\
\hline Tibetan Spaniel & 1.00 & 0.00 & 0.00 & 0.00 & {$[9,18]$} \\
\hline Tibetan Terrier & $0.46-0.53$ & 0.35 & $0.12-0.19$ & $0.29-0.36$ & {$[9,18]$} \\
\hline Toy Fox Terrier & 1.00 & 0.00 & 0.00 & 0.00 & {$[9,18]$} \\
\hline Treeing Walker Coonhound & 0.95 & 0.00 & 0.05 & 0.05 & [9] \\
\hline Vizsla & 1.00 & 0.00 & 0.00 & 0.00 & {$[9,18]$} \\
\hline Volpino Italiano & 1.00 & 0.00 & 0.00 & 0.00 & [9] \\
\hline Wachtelhund (German Spaniel) & 0.00 & 1.00 & 0.00 & 0.50 & [9] \\
\hline Weimaraner & 1.00 & 0.00 & 0.00 & 0.00 & {$[9,18]$} \\
\hline Welsh Springer Spaniel & 1.00 & 0.00 & 0.00 & 0.00 & {$[9,18]$} \\
\hline Welsh Terrier & $0.56-0.57$ & 0.35 & $0.08-0.10$ & $0.26-0.27$ & {$[9,18]$} \\
\hline West Highland White Terrier & 1.00 & 0.00 & 0.00 & 0.00 & {$[9,18]$} \\
\hline Whippet & 1.00 & 0.00 & 0.00 & 0.00 & {$[9,18]$} \\
\hline Wirehaired Pointing Griffon & 1.00 & 0.00 & 0.00 & 0.00 & {$[9,18]$} \\
\hline Wire Fox Terrier & 0.01 & 0.09 & 0.90 & 0.94 & [9] \\
\hline Yorkshire Terrier & 0.75 & 0.25 & 0.00 & 0.13 & [9] \\
\hline hybrid/mix-breed & $0.41-0.44$ & $0.11-0.16$ & $0.39-0.48$ & $0.48-0 . .53$ & {$[9,18]$} \\
\hline
\end{tabular}

\section{Conclusion}

The SOD1:c. $118 G>A$ mutation was common in the tested group of dogs; therefore, this group is suitable for a follow-up assessment of the development and progression of CDM. A case-control study on animals with evidence of sensitivity to infectious myelopathy could also provide new insights into disease pathogenesis.

\section{Authors' Contributions}

AM: Conducted research in the laboratory with support from GSZ and ALH. JI: Designed the research and drafted the manuscript with support from $\mathrm{AK}$ and AM. CDV: Collected the samples for this study. AK: Collected data and conducted genetic analysis. AM, JI, NT, and MG: Reviewed and updated the manuscript. All authors read and approved the final manuscript.

\section{Acknowledgments}

The authors declare that they did not receive any funds for this study. The authors would like to acknowledge School of Animal Biosciences, Department of Animal Science, Laboratory of Anatomy and Physiology of Farm Animals, Agricultural University of Athens, Athens, Greece, for providing necessary facilities for the study.

\section{Competing Interests}

The authors declare that they have no competing interests.

\section{Publisher's Note}

Veterinary World remains neutral with regard to jurisdictional claims in published institutional affiliation.

\section{References}

1. Averill, D.R. (1973) Degenerative myelopathy in the aging German Shepherd dog: Clinical and pathologic findings. $J$. Am. Vet. Med. Assoc., 162(12): 1045-1051.

2. Awano, T., Johnson, G.S., Wade, C.M., Katz, M.L., Taylor, J.F., Perloski, M., Biagi, T., Baranowska, I., Long, S., March, P.A., Olby, N.J., Shelton, G.D., Khan, S., O'Brien, D.P., Lindblad-Toh, K. and Coates, J.R. (2009) Genome-wide association analysis reveals a SOD1 mutation in canine degenerative myelopathy that resembles amyotrophic lateral sclerosis. Proc. Natl. Acad. Sci., 106(8): 2794-2799.

3. Shelton, G.D., Johnson, G.C., O’Brien, D.P., Katz, M.L., Pesayco, J.P., Chang, B.J., Mizisin, A.P. and Coates, J.R. (2012) Degenerative myelopathy associated with a missense mutation in the superoxide dismutase 1 (SOD1) gene progresses to peripheral neuropathy in Pembroke welsh corgis and Boxers. J. Neurol. Sci., 318(1-2): 55-64.

4. Mayberry, K. and Carithers, R.W. (1975) Degenerative myelopathy in the German shepherd. Iowa State Univ. Vet., 37(1): 7.

5. Braund, K.G. and Vandevelde, M. (1978) German Shepherd dog myelopathy-a morphologic and morphometric study. Am. J. Vet. Res., 39(8): 1309-1315.

6. Chang, H.S., Kamishina, H., Mizukami, K., Momoi, Y., Katayama, M., Rahman, M.M., Uddin, M.M., Yabuki, A., Kohyama, M. and Yamato, O. (2013) Genotyping assays for the canine degenerative Myelopathy-Associated c. $118 \mathrm{G}>\mathrm{A}$ 
(p.E40K) mutation of the SOD1 gene using conventional and real-time PCR methods: A high prevalence in the Pembroke welsh corgi breed in Japan. J. Vet. Sci., 75(6): 795-798.

7. Capucchio, M.T., Spalenza, V., Biasibetti, E., Bottero, M.T., Rasero, R., Dalmasso, A. and Sacchi, P. (2014) Degenerative myelopathy in German Shepherd dog: Comparison of two molecular assays for the identification of the SOD1: C.118G $>$ A mutation. Mol. Biol. Rep., 41(2): 665-670.

8. Holder, A.L., Price, J.A., Adams, J.P., Volk, H.A. and Catchpole, B. (2014) A retrospective study of the prevalence of the canine degenerative myelopathy associated superoxide dismutase 1 mutation (SOD1: C.118G $>$ A) in a referral population of German Shepherd dogs from the UK. Canine Genet. Epidemiol., 1(1): 10.

9. Zeng, R., Coates, J.R., Johnson, G.C., Hansen, L., Awano, T., Kolicheski, A., Ivansson, E., Perloski, M., Lindblad-Toh, K., O’Brien, D.P., Guo, J., Katz, M.L. and Johnson, G.S. (2014) Breed distribution of SOD1 alleles previously associated with canine degenerative myelopathy. J. Vet. Intern. Med., 28(2): 515-521.

10. Neeves, J. and Granger, N. (2015) An update on degenerative myelopathy in dogs. Companion Anim., 20(7): 408-412.

11. Alfahad, T. and Nath, A. (2013) Retroviruses and amyotrophic lateral sclerosis. Antiviral Res., 99(2): 180-187.

12. Nardone, R., Höller, Y., Taylor, A.C., Lochner, P., Tezzon, F., Golaszewski, S., Brigo, F. and Trinka, E. (2016) Canine degenerative myelopathy: A model of human amyotrophic lateral sclerosis. Zoology, 119(1): 64-73.

13. Garson, J.A., Usher, L., Al-Chalabi, A., Huggett, J., Day, E.F. and McCormick, A.L. (2019) Quantitative analysis of human endogenous retrovirus-K transcripts in postmortem premotor cortex fails to confirm elevated expression of HERV-K RNA in amyotrophic lateral sclerosis. Acta Neuropathol. Commun., 7(1): 45.

14. Indo, H.P., Yen, H.C., Nakanishi, I., Matsumoto, K.I., Tamura, M., Nagano, Y., Matsui, H., Gusev, O., Cornette, R., Okuda, T., Minamiyama, Y., Ichikawa, H., Suenaga, S., Oki, M., Sato, T., Ozawa, T., St Clair, D.K. and Majima, H.J. (2015) A mitochondrial superoxide theory for oxidative stress diseases and aging. J. Clin. Biochem. Nutr., 56(1): $1-7$.

15. Chang, R.C., Parakh, S., Coates, J.R., Long, S. and Atkin, J.D. (2019) Protein disulphide isomerase is associated with mutant SOD1 in canine degenerative myelopathy. Neuroreport, 30(1): 8-13.

16. Raymond, M. and Rousset, F. (1995) GENEPOP (Version 1.2): Population genetics software for exact tests and ecumenicism. J. Hered., 86(3): 248-249.

17. Weir, B.S. and Cockerham, C.C. (1984) Estimating F-statistics for the analysis of population structure. Evolution, 38(6): 1358-1370.

18. Coates, J.R., Lindblad-Toh, K., Wade, C. and Johnson, G.S. (2009) Prediction and Diagnosis of Canine Degenerative Myelopathy, United States Patent Pub. No. US 2009/0239225 A1. Available from http://www.freepatentsonline.com/ y2009/0239225.html. Retrieved on 22-05-2020.

19. Beckers, E., van Poucke, M., Ronsyn, L. and Peelman, L. (2016) Frequency estimation of disease-causing mutations in the Belgian population of some dog breeds, part 1: Shepherds. Vlaams Diergeneeskd. Tijdschr., 85(4): 175-184.

20. Ayala-Valdovinos, M.A., Gomez-Fernandez,A.A., DuifhuisRivera, T., Aparicio-Cid, E.A., Sánchez-Chiprés, D.R. and
Galindo-García, J. (2018) Frequency of canine degenerative myelopathy SOD1: C.118G $>$ A mutation in 22 dog breeds in Guadalajara, Mexico. Rev. Colomb. Cienc. Pecu., 31(2): 150-154.

21. Broeckx, B.J., Coopman, F., Verhoeven, G.E., van Haeringen, W., van de Goor, L., Bosmans, T., Gielen, I., Saunders, J.H., Soetaert, S.S., van Bree, H., van Neste, C., van Nieuwerburgh, F., van Ryssen, B., Verelst, E., van Steendam, K. and Deforce, D. (2013) The prevalence of nine genetic disorders in a dog population from Belgium, the Netherlands and Germany. PLoS One, 8(9): e74811.

22. Mizukami, K., Yabuki, A., Kohyama, M., Kushida, K., Rahman, M.M., Uddin, M.M., Sawa, M. and Yamato, O. (2016) Molecular prevalence of multiple genetic disorders in Border collies in Japan and recommendations for genetic counseling. Vet. J., 214: 21-23.

23. Kohyama, M., Kitagawa, M., Kamishina, H., Kobatake, Y., Yabuki, A., Sawa, M., Kakita, S. and Yamato, O. (2017) Degenerative myelopathy in the Collie breed: A retrospective immunohistochemical analysis of superoxide dismutase 1 in an affected Rough Collie, and a molecular epidemiological survey of the SOD1: C.118G $>$ A mutation in Japan. J. Vet. Med. Sci., 79(2): 375-379.

24. Jakabová, D., Chlebovcová, P. and Genčík, M.(2016) A genetic study of a SOD1 missense mutation in Czechoslovakian Wolfdog. Acta Fytotech. Zootech., 19: 111-113.

25. Giannuzzi, A.P., Ricciardi, M., de Simone, A. and Gernone, F. (2017) Neurological manifestations in dogs naturally infected by Leishmania infantum: Descriptions of 10 cases and a review of the literature. J. Small Anim. Pract., 58(3): 125-138.

26. Khouri, R., Bafica, A., Silva, M., Noronha, A., Kolb, J.P., Wietzerbin, J., Barral, A., Barral-Netto, M. and van Weyenbergh, J. (2009) IFN-beta impairs superoxide-dependent parasite killing in human macrophages: Evidence for a deleterious role of SOD1 in cutaneous leishmaniasis. $J$. Immunol., 182(4): 2525-2531.

27. Vivarini, A., Pereira, R., Teixeira, K.L., CalegariSilva, T.C., Bellio, M., Laurenti, M.D., Corbett, C.E., Gomes, C.M., Soares, R.P., Silva, A.M., Silveira, F.T. and Lopes, U.G. (2011) Human cutaneous leishmaniasis: Interferon-dependent expression of double-stranded RNAdependent protein kinase (PKR) via TLR2. FASEB J., 25(12): 4162-4173.

28. Gupta, G., Oghumu, S. and Satoskar, A.R. (2013) Mechanisms of immune evasion in leishmaniasis. $A d v$. Appl. Microbiol., 82: 155-184.

29. Khouri, R., Santos, G.S., Soares, G., Costa, J.M., Barral, A., Barral-Netto, M. and Weyenbergh, J.V. (2014) SOD1 plasma level as a biomarker for therapeutic failure in cutaneous leishmaniasis. J. Infect. Dis., 210(2): 306-310.

30. Carmichael, K.P., Bienzle, D. and Mcdonnell, J.J. (2002) Feline leukemia virus-associated myelopathy in cats. Vet. Pathol., 39(5): 536-545.

31. Bergman, R.L. (2006) Miscellaneous spinal cord disorders. In: August, J.R., editor. Consultations in Feline Internal Medicine. $4^{\text {th }}$ ed. Saunders, Elsevier, St. Louis, Missouri.

32. Pardo, A.P., Gómez, M.A. and González, C.M. (2019) Cerebellar cortical degeneration associated with feline leukemia virus infection and cerebellar lymphoma in a young cat. Open Vet. J., 9(3): 246-252. 